\title{
A stabilized finite element method for the mixed wave equation in an ALE framework with application to diphthong production
}

\author{
$\underline{\text { Oriol Guasch }}^{\mathrm{a}}, \underline{\text { Marc Arnela }}^{\mathrm{a}}, \underline{\text { Ramon Codina }}^{\mathrm{b}}, \underline{\text { Hector Espinoza }}^{\mathrm{b}}$ \\ ${ }^{a}$ GTM - Grup de recerca en Tecnologies Mèdia, La Salle, Universitat Ramon Llull \\ C/ Quatre Camins 2, 08022 Barcelona, Catalonia \\ ${ }^{b}$ Universitat Politècnica de Catalunya, \\ Jordi Girona 1-3, Edifici C1, 08034 Barcelona, Catalonia
}

\begin{abstract}
Working with the wave equation in mixed rather than irreducible form allows one to directly account for both, the acoustic pressure field and the acoustic particle velocity field. Indeed, this becomes the natural option in many problems, such as those involving waves propagating in moving domains, because the equations can easily be set in an arbitrary Lagrangian-Eulerian (ALE) frame of reference. Yet, when attempting a standard Galerkin finite element solution (FEM) for them, it turns out that an inf-sup compatibility constraint has to be satisfied, which prevents from using equal interpolations for the approximated acoustic pressure and velocity fields. In this work it is proposed to resort to a subgrid scale stabilization strategy to circumvent this condition and thus facilitate code implementation. As a possible application, we address the generation of diphthongs in voice production.
\end{abstract}

Keywords: Voice production, Diphthongs, ALE mixed wave equation, Stabilized finite elements methods, Subgrid scale stabilization

\section{Introduction}

The wave equation in mixed form refers, in classical acoustics, to the continuity and momentum equations obtained after linearization of the compressible Navier-Stokes equations for an inviscid homentropic fluid. In contrast to the wave equation in irreducible form, it allows one to describe both, the propagation of acoustic pressure waves and of the acoustic particle velocity as well. This becomes of importance in many diferent topics which range from intensimetry techniques [1], acoustic wave propagation in ducts [2] or the computation of radiation impedances [3]. Moreover, the wave equation in mixed form also allows one to describe related phenomena in other areas of physics such as elastodynamics or the behavior of waves in shallow waters without convection (see e.g., [4]).

In this work we are interested in numerically solving the problem of wave propagation in moving domains. In particular, our final goal is to be able to produce diphthongs. Though many computational efforts have been placed to solve the fluid-structure problem of human phonation (see e.g., $\underline{[5,} \underline{6,} \underline{7,} \underline{8,} 9]$ ), to the best of our knowledge little has been done with regard to vocal tract acoustics in moving domains, as it is the case for diphthong or syllable generation. Generating diphthongs requires solving the wave equation in a human vocal tract that evolves from the shape, say for instance of vowel /a/, to the shape of vowel /i/, thus producing the sound /ai/. It is to be noted that for the numerical generation of vowels and diphthongs the acoustic pressure is the variable of interest, so most works to date concerning the generation of vowels directly deal with the FEM solution of the irreducible wave equation [10, 11, 12, 13, 14], or with its time Fourier counterpart, the Helmholtz equation $[15, \underline{16}, 17, \underline{18}$. Occasionally, the wave equation in mixed form

Email address: oguasch@salleurl.edu (Oriol Guasch)

$U R L:$ www.salle.url.edu/ oguasch (Oriol Guasch) 
has also been solved [19]. In fact, it will be shown that the latter becomes the most natural option when dealing with acoustic wave propagation in moving domains. In such cases the flow continuity and momentum equations have to be expressed in an Arbitrary Lagrangian Eulerian (ALE) [20, 21] frame of reference, rather than in a pure Eulerian one, and this is not straightforward for the irreducible wave equation.

From a numerical and analytical point of view, there are some important differences between the mixed wave equation and its ALE counterpart. Their FEM solutions rely on discretizing the corresponding weak forms. In the case of the variational mixed wave equation, a compatibility inf-sup condition has to be satisfied for the problem to be well-posed. This condition is not directly inherited by the standard Galerkin FEM approach and it becomes necessary to make use of different interpolation spaces for the acoustic pressure and particle velocity to attain a solution free of spurious oscillations $[22,23,24]$. Alternatively, one can resort to stabilized FEM methods, such as the residual based multiscale methods [25, 26, 27], which allows one to use the same interpolation for the acoustic pressure and particle velocity approximations [4]. A detailed numerical analysis reveals that in this case the solution becomes bounded by the problem data in a new energy norm that contains all designed stabilization terms [28]. The situation becomes more intricate for the ALE wave equation in mixed form. This equation has the same mathematical structure as the linearized modified Boussinesq equation for shallow waters with convection. Numerical experiments show that the Galerkin FEM solution for the latter exhibits strong high-frequency oscillations if equal interpolations are used for the gravity waves depth and velocity [29, 30]. Again these oscillations can be overcome if one draws on stabilization strategies $[31,32]$. In this work we will present a subgrid scale stabilization FEM approach tailored to the ALE wave equation in mixed form, following the main lines in [32]. It will be shown that the proposed strategy will allow us to use equal interpolations for the acoustic pressure and velocity, and prevent the FEM solution to blow up. It is to be noted however, that contrary to the variational mixed wave

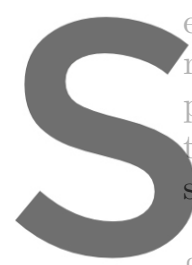
equation, little is know

mixed wave equation. Act proved showing that the the ALE mixed wave equat

simple rectangular geome

This paper is organized
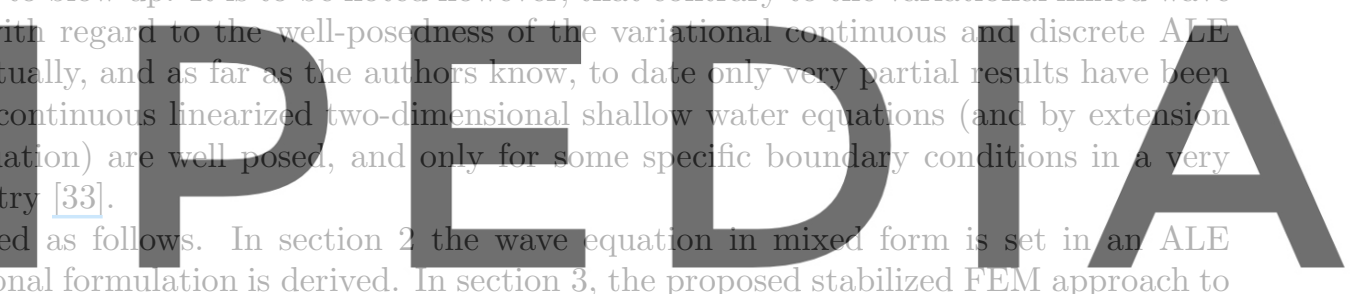

framework and its variational formulation is derived. In section 3 , the proposed stabilized FEM approach to solve the latter is presented. Special emphasis is placed on the design of the so called matrix of stabilization

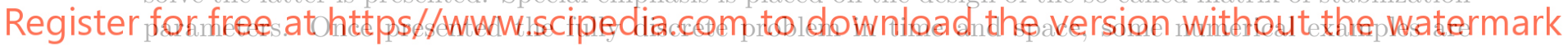
provided in section 4. First, a test is made to check the performance of the stabilized formulation. It consists of plane waves propagating in a straight duct with time varying length due to the periodic movement of the exit boundary. Second, an example consisting in the production of diphthong /ai/ is presented. Not only waves can be visualized propagating inside the vocal tract and exhibiting the right spectrogram, but also an audio file corresponding to /ai/ can be generated by collecting the pressure time evolution at a target node.

\section{Problem statement}

\subsection{The mixed wave equation in an ALE framework}

The linearized continuity and momentum equations that describe sound propagation in a stationary inviscid fluid read, in a spatial (Eulerian) frame of reference,

$$
\begin{array}{r}
\frac{1}{\rho_{0} c_{0}^{2}} \partial_{t} p+\nabla \cdot \boldsymbol{u}=Q, \\
\rho_{0} \partial_{t} \boldsymbol{u}+\nabla p=\boldsymbol{f},
\end{array}
$$

where $p(\boldsymbol{x}, t)$ stands for the acoustic pressure and $\boldsymbol{u}(\boldsymbol{x}, t)$ for the acoustic particle velocity. $Q(\boldsymbol{x}, t)$ represents a volume source distribution and $\boldsymbol{f}(\boldsymbol{x}, t)$ an external body force per unit volume. The parameters $c_{0}$ and $\rho_{0}$ respectively denote the speed of sound and the mean air density, and $\partial_{t}$ is used to denote the first order time derivative. Equation (1) is sometimes referred to as the wave equation in mixed form. Its standard 
irreducible counterpart for the acoustic pressure can be derived subtracting the divergence of (1b) from $\rho_{0}$ times the time derivative of (1a). This results in

$$
\frac{1}{c_{0}^{2}} \partial_{t t}^{2} p-\nabla^{2} p=\rho_{0} \partial_{t} Q-\nabla \cdot \boldsymbol{f}
$$

In this work we are interested in solving the mixed wave equation (1) in a domain with moving boundaries. This poses some problems to the Eulerian description of the equations given that one has to take into account the relative movement between the fluid particles and the moving domain. Moreover, dealing with the dynamic boundaries is not straightforward in the Eulerian framework and requires some special approaches such as space-time finite element methods. Yet, setting the mixed wave equation in a pure Lagrangian frame of reference is not exempt of difficulties because computationally expensive mesh rezoning becomes often necessary. An approach that provides a good balance overcoming some of the complications of both descriptions is the so called quasi-Eulerian ALE formulation (see e.g., [34] and references therein). In this case, the time derivative of any fuid property, say $g$, is expressed in a referential frame moving with the domain, while the spatial derivatives of $g$ are kept in an Eulerian frame [20, 21]. To obtain the ALE expressions for the continuity and momentum equations in (1), we proceed by replacing

$$
\partial_{t} g \leftarrow \partial_{t} g-u_{d} \cdot \nabla g
$$

in them, with $\boldsymbol{u}_{d}(\boldsymbol{x}, t)$ denoting the domain velocity. This provides the ALE wave equation in mixed form

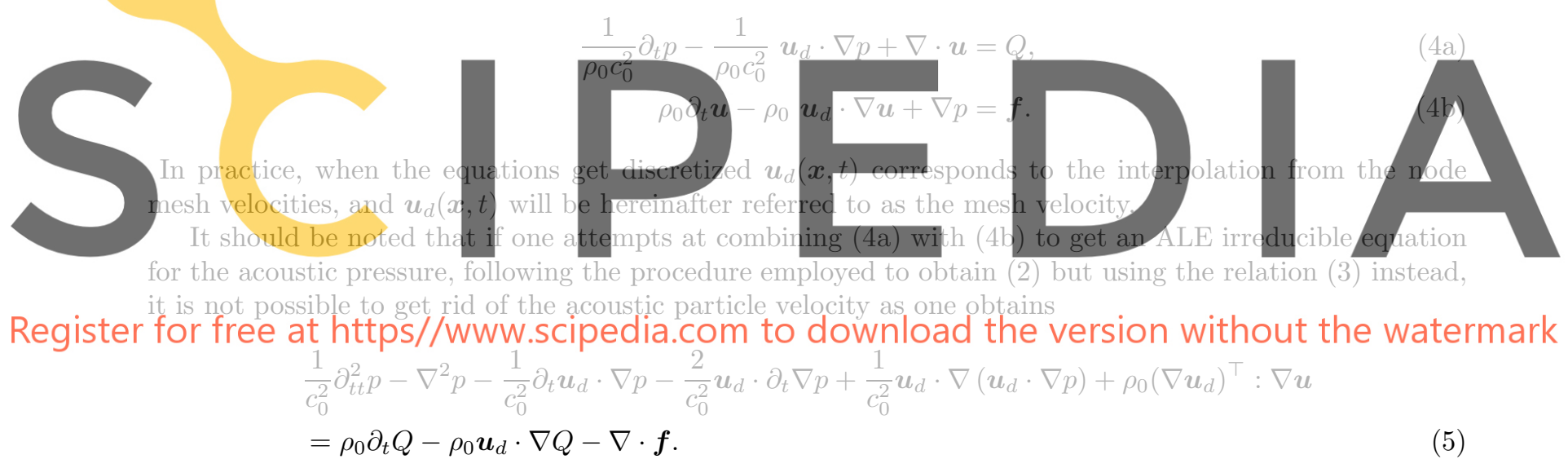

Therefore, the most natural option to deal with wave propagation in moving domains seems to be that of directly dealing with the wave equation in mixed form. Though it is possible to factorize the wave equation $(2)$ as $\left(-\frac{1}{c_{0}^{2}} \partial_{t t}^{2} p+\nabla^{2} p\right)=\left(\frac{1}{c_{0}} \partial_{t}+\nabla\right)\left(-\frac{1}{c_{0}} \partial_{t}+\nabla\right) p$, one should be prevented from substituting (3) in it. As explained at the beginning of this section, equation (2) is obtained from the mass and momentum conservation equations (1a) and (1b) expressed in an Eulerian frame of reference, not an ALE one. It would then be erroneous to directly use (3) in it.

\subsection{Variational formulation}

Let us next suppose that (4) is to be solved in a computational domain $\Omega$ with boundary $\partial \Omega$. $\partial \Omega$ is made of the union of two disjoint sets $\Gamma_{p}$ and $\Gamma_{\boldsymbol{u}}$, the pressure being prescribed on the former and the velocity on the latter. For the ease of exposition we will consider homogeneous Dirichlet conditions on them, i.e., $p=0$ on $\Gamma_{p}, \quad t>0$ and $\boldsymbol{u} \cdot \boldsymbol{n}=0$ on $\Gamma_{\boldsymbol{u}}, \quad t>0$. We also consider initial conditions $p(\boldsymbol{x}, 0)=p^{0}(\boldsymbol{x})$ and $\boldsymbol{u}(\boldsymbol{x}, 0)=\boldsymbol{u}^{0}(\boldsymbol{x})$ in $\Omega$.

The weak formulation of (4) is found as usual by multiplying (4a) with a scalar test function $q$, (4b) with a vector test function $\boldsymbol{v}$, and integrating over the computational domain $\Omega$. If we denote the integral between two arbitrary functions $f$ and $g$ as $(f, g):=\int_{\Omega} f g d \Omega$, assuming $\boldsymbol{u}_{d}$ to be smooth enough, the variational 
problem can be formulated as that of finding $p \in C^{1}\left([0, T], L^{2}\right) \cap C^{0}\left([0, T], V_{p}\right)$ and $\boldsymbol{u} \in C^{1}\left([0, T], \boldsymbol{L}^{2}\right) \cap$ $C^{0}\left([0, T], \boldsymbol{V}_{\boldsymbol{u}}\right)$ such that

$$
\begin{aligned}
\frac{1}{\rho_{0} c_{0}^{2}}\left(\partial_{t} p, q\right)-\frac{1}{\rho_{0} c_{0}^{2}}\left(\boldsymbol{u}_{d} \cdot \nabla p, q\right)+(\nabla \cdot \boldsymbol{u}, q)=(Q, q) & \forall q \in V_{p}, \\
\rho_{0}\left(\partial_{t} \boldsymbol{u}, \boldsymbol{v}\right)-\rho_{0}\left(\boldsymbol{u}_{d} \cdot \nabla \boldsymbol{u}, \boldsymbol{v}\right)+(\nabla p, \boldsymbol{v})=(\boldsymbol{f}, \boldsymbol{v}) & \forall \boldsymbol{v} \in \boldsymbol{V}_{\boldsymbol{u}},
\end{aligned}
$$

with $V_{p}=\left\{p \in H^{1}(\Omega) \mid p=0\right.$ on $\left.\Gamma_{p}\right\}$ and $\boldsymbol{V}_{\boldsymbol{u}}=\left\{\boldsymbol{v} \in \boldsymbol{L}^{2}(\Omega) \mid \nabla \cdot \boldsymbol{v} \in L^{2}(\Omega), \boldsymbol{u}_{d} \cdot \nabla \boldsymbol{v} \in \boldsymbol{L}^{2}(\Omega), \boldsymbol{v} \cdot \boldsymbol{n}=0\right.$ on $\left.\Gamma_{\boldsymbol{u}}\right\}$. Note that $(\cdot, \cdot)$ may either represent a scalar product in $L^{2}(\Omega)$ (also in $\boldsymbol{L}^{2}(\Omega)$ ), or a duality pairing depending e.g., on the functional setting of the forcing terms.

With regard to the variational formulation in (6), it should be noted that:

- The Dirichlet boundary conditions (homogeneous in this case) for the acoustic pressure and velocity in (6) are to be strongly imposed on $\partial \Omega$. One could alternatively weakly impose the pressure and strongly impose the velocity integrating by parts the term $(\nabla p, \boldsymbol{v})$, or viceversa, integrating the term $(\nabla \cdot u, q)$. If the ALE terms are missing $\left(u_{d}=0\right)$, the standard wave equation in mixed form is recovered. Integration of the terms $(\nabla p, v)$ or $(\nabla \cdot u, q)$ noticeably changes in that case the functional framework where the solution for the acoustic pressure and particle velocity are to be found. When attempting a numerical approximation to the corresponding variational forms by means of FEM, this allows one either to obtain the same degree of accuracy for the pressure and the velocity, or to favor one of them at the expense of the other [28].

- As quoted in the Introduction, the ALE wave equation in mixed form, (6), closely ressembles the linearized modified Boussinesq equation for shallow waters. The Galerkin FEM solution for the latter
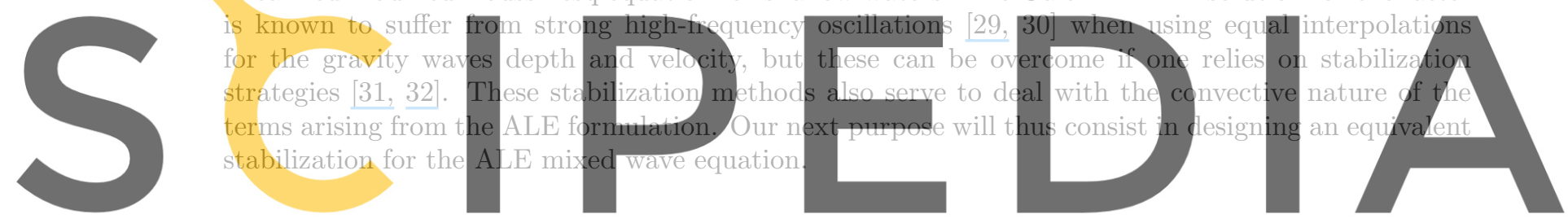

3. Subgrid scale stabilized finite element method

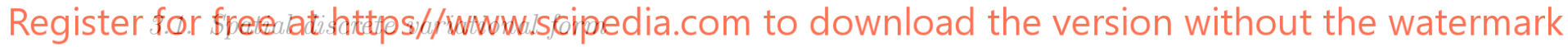

In this section we will present a subgrid scale stabilization FEM approach for the ALE wave equation in mixed form, based on the variational multiscale method $[25,26]$. This will avoid locking effects when using the same interpolations for the acoustic pressure and velocity, as well as accounting for the treatment of the convective terms arising from the ALE formulation. To do so we will closely follow the main lines in previous works on stabilization of the wave equation in mixed form [4] and on the modified Boussinesq equations [32].

First of all, let us rewrite problem (4) in compact matrix form as

$$
\boldsymbol{\mu} \partial_{t} \boldsymbol{U}+\boldsymbol{A}_{i} \partial_{i} \boldsymbol{U}=\boldsymbol{F},
$$

where the summation convention over repeated indexes is assumed when they run from 1 to the number of spatial dimensions, which we assume to be 3 in what follows. The following identifications have been made,

$$
\begin{aligned}
& \boldsymbol{U}=\left(\begin{array}{c}
p \\
u_{1} \\
u_{2} \\
u_{3}
\end{array}\right), \boldsymbol{F}=\left(\begin{array}{c}
Q \\
f_{1} \\
f_{2} \\
f_{3}
\end{array}\right), \boldsymbol{\mu}=\left(\begin{array}{cccc}
\mu_{p} & 0 & 0 & 0 \\
0 & \mu_{\boldsymbol{u}} & 0 & 0 \\
0 & 0 & \mu_{\boldsymbol{u}} & 0 \\
0 & 0 & 0 & \mu_{\boldsymbol{u}}
\end{array}\right), \boldsymbol{A}_{1}=\left(\begin{array}{cccc}
-\mu_{p} u_{d 1} & 1 & 0 & 0 \\
1 & -\mu_{\boldsymbol{u}} u_{d 1} & 0 & 0 \\
0 & 0 & -\mu_{\boldsymbol{u}} u_{d 1} & 0 \\
0 & 0 & 0 & -\mu_{\boldsymbol{u}} u_{d 1}
\end{array}\right), \\
& \boldsymbol{A}_{2}=\left(\begin{array}{cccc}
-\mu_{p} u_{d 2} & 0 & 1 & 0 \\
0 & -\mu_{\boldsymbol{u}} u_{d 2} & 0 & 0 \\
1 & 0 & -\mu_{\boldsymbol{u}} u_{d 2} & 0 \\
0 & 0 & 0 & -\mu_{\boldsymbol{u}} u_{d 2}
\end{array}\right), \boldsymbol{A}_{3}=\left(\begin{array}{cccc}
-\mu_{p} u_{d 3} & 0 & 0 & 1 \\
0 & -\mu_{\boldsymbol{u}} u_{d 3} & 0 & 0 \\
0 & 0 & -\mu_{\boldsymbol{u}} u_{d 3} & 0 \\
1 & 0 & 0 & -\mu_{\boldsymbol{u}} u_{d 3}
\end{array}\right) \text {. }
\end{aligned}
$$


To ease the notation and to facilitate comparisons with the preceding work in [4] as well, we have introduced the parameters $\mu_{p} \equiv\left(\rho_{0} c_{0}^{2}\right)^{-1}$ and $\mu_{\boldsymbol{u}} \equiv \rho_{0}$ which fulfill $c_{0}=\left(\mu_{p} \mu_{\boldsymbol{u}}\right)^{-1 / 2} \cdot u_{d i}, i=1,2,3$, denote the components of the mesh velocity vector at each point, $\boldsymbol{u}_{d}=\left(u_{d 1}, u_{d 2}, u_{d 3}\right)^{\top}$.

The variational formulation of (7) is found as usual, multiplying by a test function $\boldsymbol{V}$ and integrating over the computational domain. If we define the spaces $\mathcal{L} \equiv L^{2} \times \boldsymbol{L}^{2}$ and $\mathcal{V} \equiv V_{p} \times \boldsymbol{V}_{\boldsymbol{u}}$, the problem becomes that of finding $\boldsymbol{U} \in C^{1}([0, T], \mathcal{L}) \cap C^{0}([0, T], \mathcal{V})$ such that

$$
\left(\boldsymbol{\mu} \partial_{t} \boldsymbol{U}, \boldsymbol{V}\right)+\left(\boldsymbol{A}_{i} \partial_{i} \boldsymbol{U}, V\right)=(\boldsymbol{F}, \boldsymbol{V}) \quad \forall \boldsymbol{V} \in \mathcal{L} \cap \mathcal{V}
$$

The discretized conforming Galerkin FEM approach to the problem then consists in finding a finite element solution $\boldsymbol{U}_{h} \in C^{1}\left([0, T], \mathcal{L}_{h} \subset \mathcal{L}\right) \cap C^{0}\left([0, T], \mathcal{V}_{h} \subset \mathcal{V}\right)$ that satisfies

$$
\left(\boldsymbol{\mu} \partial_{t} \boldsymbol{U}_{h}, \boldsymbol{V}_{h}\right)+\left(\boldsymbol{A}_{i} \partial_{i} \boldsymbol{U}_{h}, \boldsymbol{V}_{h}\right)=\left(\boldsymbol{F}, \boldsymbol{V}_{h}\right) \quad \forall \boldsymbol{V}_{h} \in \mathcal{L}_{h} \cap \mathcal{V}_{h}
$$

Here, $\mathcal{L}_{h} \cap \mathcal{V}_{h}$ is a finite space constructed from a finite element partition $\left\{\Omega^{e}\right\}$ of $\Omega$, with index $e$ ranging from 1 to the number of elements $n_{e l}$. Given the aforementioned problems with the Galerkin FEM formulation (10), a variational multiscale stabilization approach is next introduced. This consists in splitting the exact solution $\boldsymbol{U}$ into a finite element component $\boldsymbol{U}_{h}$ that can be resolved by the computational mesh, plus a subscale $\boldsymbol{U}^{\prime}$ which cannot be computed, and has to be somehow modeled. Substitution of $\boldsymbol{U}=\boldsymbol{U}_{h}+\boldsymbol{U}^{\prime}$ into (10) yields two equations; the first one accounting for the large solvable scales is given by

$$
\left(\boldsymbol{\mu} \partial_{t} \boldsymbol{U}_{h}, \boldsymbol{V}_{h}\right)+\left(\boldsymbol{\mu} \partial_{t} \boldsymbol{U}^{\prime}, \boldsymbol{V}_{h}\right)+\left(\boldsymbol{A}_{i} \partial_{i} \boldsymbol{U}_{h}, \boldsymbol{V}_{h}\right)+\left(\boldsymbol{A}_{i} \partial_{i} \boldsymbol{U}^{\prime}, \boldsymbol{V}_{h}\right)=\left(\boldsymbol{F}, \boldsymbol{V}_{h}\right) .
$$

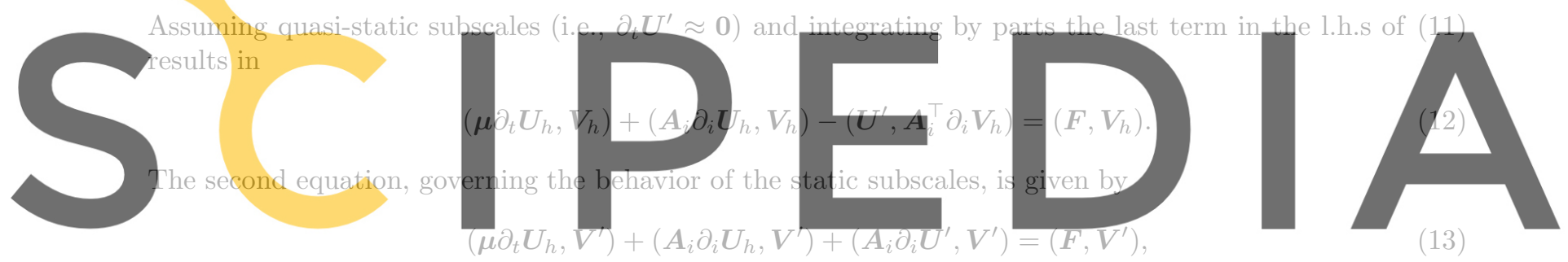

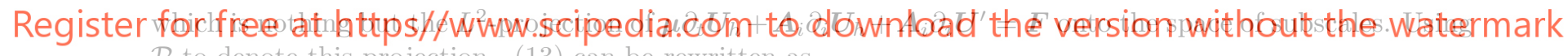
$\mathcal{P}$ to denote this projection, (13) can be rewritten as

$$
\mathcal{P}\left(\boldsymbol{A}_{i} \partial_{i} \bar{U}^{\prime}\right)=\mathcal{P}\left[\boldsymbol{F}-\left(\partial_{t} \bar{U}_{h}+\boldsymbol{A}_{i} \partial_{i} \bar{U}_{h}\right)\right]=: \boldsymbol{R}_{h}
$$

where we have defined the residual $\boldsymbol{R}_{h}$ of the finite element approximation onto the subscale space in the last equality. As said, (14) is an equation for the subscales $\boldsymbol{U}^{\prime}$ whose solution is unknown and has to be modeled. A reasonable approximation is that of taking [4, 32] $\mathcal{P}\left(\boldsymbol{A}_{i} \partial_{i} \boldsymbol{U}^{\prime}\right) \approx \boldsymbol{\tau}^{-1} \boldsymbol{U}^{\prime}$, so that from (14) $\boldsymbol{U}^{\prime}=\boldsymbol{\tau} \boldsymbol{R}_{h}$, with $\boldsymbol{\tau}$ standing for a symmetric, positive-definite matrix of stabilization parameters to be determined. Substituting $\boldsymbol{U}^{\prime}=\boldsymbol{\tau} \boldsymbol{R}_{h}$ into the large scale equation (12) yields

$$
\left(\boldsymbol{\mu} \partial_{t} \boldsymbol{U}_{h}, \boldsymbol{V}_{h}\right)+\left(\boldsymbol{A}_{i} \partial_{i} \boldsymbol{U}_{h}, \boldsymbol{V}_{h}\right)+\sum_{e=1}^{n_{e l}}\left(\boldsymbol{A}_{i}^{\top} \partial_{i} \boldsymbol{V}_{h}, \boldsymbol{\tau} \mathcal{P}\left[\left(\partial_{t} \boldsymbol{U}_{h}+\boldsymbol{A}_{i} \partial_{i} \boldsymbol{U}_{h}\right)-\boldsymbol{F}\right]\right)_{\Omega_{e}}=\left(\boldsymbol{F}, \boldsymbol{V}_{h}\right)
$$

The first two terms in (15) correspond to the standard Galerkin FEM approach in (10), whereas the third one is the additional stabilization term that facilitates using equal interpolations for the acoustic pressure and acoustic particle velocity. Concerning the stabilized variational formulation we note that

- There exist different options for the projection operator $\mathcal{P}$ in the above expression. In the so called OSS (Orthogonal Subgrid Scale) method [27, 35], the subscales are assumed to lay in a space orthogonal to the finite element space so that $\mathcal{P}$ can be computed as $\mathcal{P}=\boldsymbol{I}-\Pi_{h}, \Pi_{h}$ being the $L^{2}$-projection onto the finite element space, and $\boldsymbol{I}$ the identity matrix. Alternatively, in the more classical ASGS (Algebraic Subgrid Scale) approach we simply take $\mathcal{P}=\boldsymbol{I}$ over the space of finite element residuals. 
- The approximation for the subscales is local and these are assumed to vanish at the interelemental boundaries. The integrals over the computational domain $\Omega$ that contain subscales are evaluated as a summation of integrals over finite element domains $\Omega_{e}$.

- The integrals in the above variational formulations contain products of vector functions having the dimensions $\left[\boldsymbol{U}^{\top} \boldsymbol{F}\right]$ ([.] is used to denote a dimensional group). Given that $\boldsymbol{U}^{\top} \boldsymbol{F}=p Q+u_{1} f_{1}+u_{2} f_{2}+$ $u_{3} f_{3}$ we require that $[p Q]=\left[u_{i} f_{i}\right]$. This is the case for the ALE mixed wave equation as it can be readily be checked that $[p Q]=\left[u_{i} f_{i}\right]=M L T^{-3}, M, L$ and $T$ respectively standing for dimensions of mass, length and time. However, in most equations vector products of the type $\boldsymbol{U}^{\top} \boldsymbol{F}$ are not well defined and a rescaling of the differential equation becomes necessary for the variational form in the compact matrix form to make sense $[4,32]$.

- To complete the weak formulation (15) it only remains finding an expression for the stabilization matrix $\boldsymbol{\tau}$. This will be the subject of the next subsection.

\subsection{The matrix $\tau$ of stabilization parameters}

The procedure to find the stabilization matrix $\tau$, to be described below, involves dealing with the norms of the residual vector, which has dimensions of force, and of the subscale velocity vector. However, we note that contrary to $U^{\top} \boldsymbol{F}$, products like $\boldsymbol{F}^{\top} \boldsymbol{F}$ or $\boldsymbol{U}^{\top} \boldsymbol{U}$ are not dimensionally well defined. For instance, $\boldsymbol{F}^{\top} \boldsymbol{F}=Q^{2}+f_{1}^{2}+f_{2}^{2}+f_{3}^{2}$ but $\left[Q^{2}\right] \neq\left[f_{i}^{2}\right]$. Analogously $\boldsymbol{U}^{\top} \boldsymbol{U}=p^{2}+u_{1}^{2}+u_{2}^{2}+u_{3}^{2}$ and again, obviously, $\left[p^{2}\right] \neq\left[u_{i}^{2}\right]$. To correctly define these products we can introduce the weighting matrix $\underline{[4]} \boldsymbol{M}$,

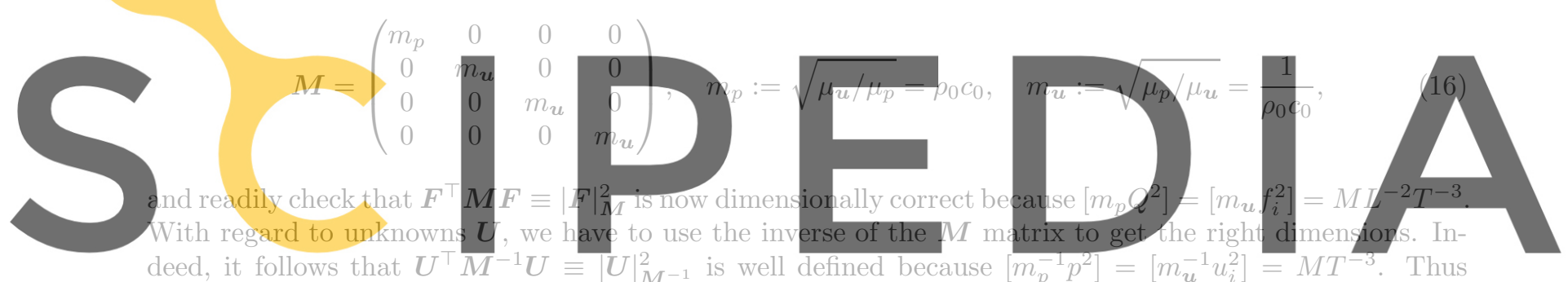

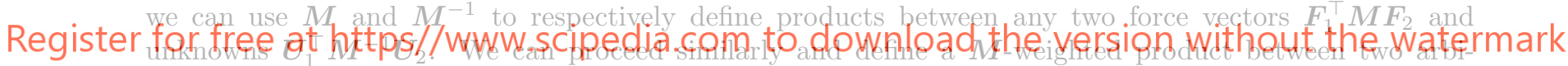

trary matrices $B_{1}$ and $B_{2}$ as $B_{1}^{\top} M B_{2}$. The squared $M$-pointwise norm of a matrix $B$ will be given by $|B|_{M \mathbb{M}}^{2}=\sup \left\{\boldsymbol{X}^{\top} B^{\top} M B \boldsymbol{X}\right\}, \forall \boldsymbol{X} ;|\boldsymbol{X}|_{\mathbb{M}^{-1}}=1$. Equipped with the weighting matrix $M$ and for regular enough force vector functions, we can also define the following vector function scalar products,

$$
(\boldsymbol{U}, \boldsymbol{F})=\int_{\Omega} \boldsymbol{U}^{\top} \boldsymbol{F} d \Omega,\left(\boldsymbol{F}_{1}, \boldsymbol{F}_{2}\right)_{\boldsymbol{M}}:=\int_{\Omega} \boldsymbol{F}_{1}^{\top} \boldsymbol{M} \boldsymbol{F}_{2} d \Omega,\left(\boldsymbol{U}_{1}, \boldsymbol{U}_{2}\right)_{\boldsymbol{M}^{-1}}:=\int_{\Omega} \boldsymbol{U}_{1}^{\top} \boldsymbol{M}^{-1} \boldsymbol{U}_{2} d \Omega,
$$

whose induced norms will be denoted by $\|\cdot\|,\|\cdot\|_{M}$ and $\|\cdot\|_{M^{-1}}$.

A suitable option to find the matrix of stabilization parameters $\boldsymbol{\tau}$ is based on a Fourier analysis of the equation for the subscales (14). Let use a hat symbol to denote Fourier transformed functions. Transforming (14) to the wave number domain allows us to bound the norm of the Fourier transformed residual $\hat{\boldsymbol{R}}_{h}$ as $\underline{\underline{4}, \underline{32]}}$

$$
\left\|\hat{\boldsymbol{R}}_{h}\right\|_{\boldsymbol{M}}^{2} \leq\left|-\mathrm{i} \frac{1}{h} k_{j}^{0} \boldsymbol{A}_{j}\right|_{\boldsymbol{M}}^{2}\left\|\hat{\boldsymbol{U}}^{\prime}\right\|_{\boldsymbol{M}^{-1}}^{2},
$$

with $k_{j}^{0}$ standing for particular dimensionless wave number components that arise from the application of the mean value theorem and that, in practice, will be treated as algorithmic constants (see below). On the other hand, from the Fourier transform of the approximation for the subscales $\boldsymbol{U}^{\prime}=\boldsymbol{\tau} \boldsymbol{R}_{h}$, we can get the bound

$$
\left\|\hat{\boldsymbol{R}}_{h}\right\|_{\boldsymbol{M}}^{2} \leq\left|\boldsymbol{\tau}^{-1}\right|_{\boldsymbol{M}}^{2}\left\|\hat{\boldsymbol{U}}^{\prime}\right\|_{\boldsymbol{M}^{-1}}^{2} .
$$


Therefore, (18) and (19) provide a means to get the stabilization matrix $\boldsymbol{\tau}$ by equating $\left|\boldsymbol{\tau}^{-1}\right|_{\boldsymbol{M}}^{2}$ with $\mid-$ $\left.\mathrm{i} \frac{1}{h} k_{j}^{0} \boldsymbol{A}_{j}\right|_{\boldsymbol{M}} ^{2}$, i.e.,

$$
\sup _{|\boldsymbol{X}|_{M^{-1}=1}} \boldsymbol{X}^{\top}\left(\boldsymbol{\tau}^{-1} \boldsymbol{M} \boldsymbol{\tau}^{-1}\right) \boldsymbol{X}=\sup _{|\boldsymbol{X}|_{M^{-1}=1}} \boldsymbol{X}^{\top}\left(\frac{1}{h^{2}} k_{i}^{0} k_{j}^{0} \boldsymbol{A}_{i}^{\top} \boldsymbol{M} \boldsymbol{A}_{j}\right) \boldsymbol{X}
$$

In practice we can implement the above condition by computing the spectra of matrices $\boldsymbol{\tau}^{-1} \boldsymbol{M} \boldsymbol{\tau}^{-1}$ and $\frac{1}{h^{2}} k_{i}^{0} k_{j}^{0} \boldsymbol{A}_{i}^{\top} \boldsymbol{M} \boldsymbol{A}_{j}$ with respect to matrix $\boldsymbol{M}^{-1}$, and then imposing their spectral radii to be the same.

Taking into account the definitions for $\boldsymbol{A}_{i}$ in (8) and for the scaling matrix $\boldsymbol{M}$ in (16), we get for the problem at hand,

$$
\begin{aligned}
& \frac{1}{h^{2}} k_{i}^{0} k_{j}^{0} \boldsymbol{A}_{i}^{\top} \boldsymbol{M} \boldsymbol{A}_{j}=\frac{1}{h^{2}} \times \\
& \left(\begin{array}{cccc}
m_{\boldsymbol{u}}\left|\boldsymbol{k}^{0}\right|^{2}+\beta_{p}\left|\boldsymbol{k}^{0} \cdot \boldsymbol{u}_{d}\right|^{2} & -\alpha k_{1}^{0}\left(\boldsymbol{k}^{0} \cdot \boldsymbol{u}_{d}\right) & -\alpha k_{2}^{0}\left(\boldsymbol{k}^{0} \cdot \boldsymbol{u}_{d}\right) & -\alpha k_{3}^{0}\left(\boldsymbol{k}^{0} \cdot \boldsymbol{u}_{d}\right) \\
-\alpha k_{1}^{0}\left(k^{0} \cdot u_{d}\right) & m_{p}\left(k_{1}^{0}\right)^{2}+\beta_{u}\left|k^{0} \cdot u_{d}\right|^{2} & k_{1}^{0} k_{2}^{0} m_{p} & k_{1}^{0} k_{3}^{0} m_{p} \\
-\alpha k_{2}^{0}\left(k^{0} \cdot u_{d}\right) & k_{1}^{0} k_{2}^{0} m_{p} & m_{p}\left(k_{2}^{0}\right)^{2}+\beta_{u}\left|k^{0} \cdot u_{d}\right|^{2} & k_{2}^{0} k_{3}^{0} m_{p} \\
-\alpha k_{3}^{0}\left(k^{0} \cdot u_{d}\right) & k_{1}^{0} k_{3}^{0} m_{p} & k_{2}^{0} k_{3}^{0} m_{p} & m_{p}\left(k_{3}^{0}\right)^{2}+\beta_{u}\left|k^{0} \cdot u_{d}\right|^{2}
\end{array}\right) \\
& \text { with } \\
& \alpha \equiv\left(m_{p} \mu_{p}+m_{u} \mu_{u}\right), \quad \beta_{p} \equiv m_{p} \mu_{p}^{2}, \quad \beta_{u} \equiv m_{u} \mu_{u}^{2} .
\end{aligned}
$$

Let us denote the spectrum of the generalized eigenvalue problem $B U=\lambda M^{-1} U, \lambda$ being an eigenvalue,

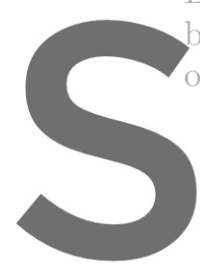
$\operatorname{Spec}_{M^{-1}}(B)$, and its at and is given by

$\operatorname{Spec}_{M-1}\left(k_{i}^{0} k_{j}^{0} A_{i}^{\top} M\right.$
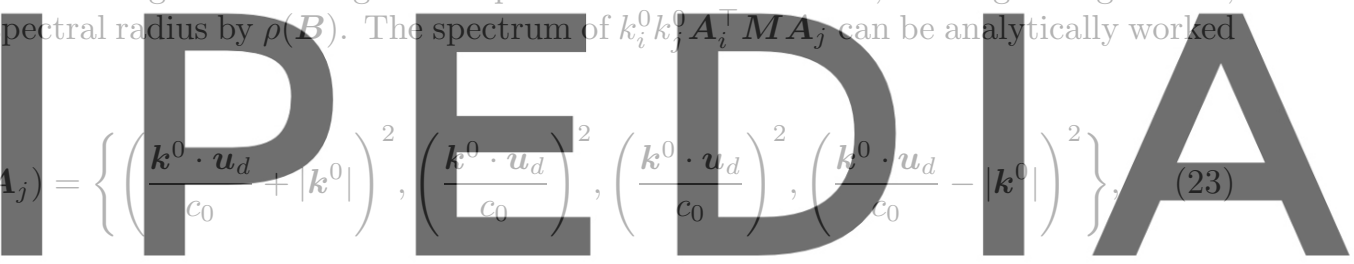

where, as seen, the eigenvalue $c_{0}^{-2}\left(k^{0} \cdot u_{d}\right)^{2}$ has multiplicity 2. As observed, the spectral radius of $k_{i}^{0} k_{j}^{0} \boldsymbol{A}_{i}^{\top} \boldsymbol{M} \boldsymbol{A}_{j}$

Registeriforvfpee at https//www.scipedia.com to download the version without the watermark

$$
\rho\left(k_{i}^{0} k_{j}^{0} \boldsymbol{A}_{i}^{\top} M A_{j}\right)=\left(\frac{k^{0} \cdot u_{d}}{c_{0}}+\left|k^{0}\right|\right)^{2} \equiv\left(\frac{C_{1}\left|u_{d}\right|}{c_{0}}+C_{2}\right)^{2} .
$$

Since $\boldsymbol{k}^{0}$ is an unknown wave number, in the r.h.s equivalence we have taken $\boldsymbol{k}^{0} \cdot \boldsymbol{u}_{d}=\left|\boldsymbol{k}^{0}\right|\left|\boldsymbol{u}_{d}\right| \cos \theta \equiv C_{1}\left|\boldsymbol{u}_{d}\right|$ and $\left|\boldsymbol{k}^{0}\right| \equiv C_{2}, C_{1}, C_{2}$ being constants that should be determined from numerical experiments.

In order to get a simple expression for the stabilization matrix we may take it to be diagonal, $\boldsymbol{\tau}=$ $\operatorname{diag}\left(\tau_{p}, \tau_{\boldsymbol{u}}, \tau_{\boldsymbol{u}}, \tau_{\boldsymbol{u}}\right)$. Given that the scaling matrix $\boldsymbol{M}$ in (16) is also diagonal, it follows that

$$
\operatorname{Spec}_{\boldsymbol{M}^{-1}}\left(\boldsymbol{\tau}^{-1} \boldsymbol{M} \boldsymbol{\tau}^{-1}\right)=\left\{\left(\frac{m_{p}}{\tau_{p}}\right)^{2},\left(\frac{m_{\boldsymbol{u}}}{\tau_{\boldsymbol{u}}}\right)^{2},\left(\frac{m_{\boldsymbol{u}}}{\tau_{\boldsymbol{u}}}\right)^{2},\left(\frac{m_{\boldsymbol{u}}}{\tau_{\boldsymbol{u}}}\right)^{2}\right\} .
$$

As we want to fulfill the condition

$$
\rho\left(\boldsymbol{\tau}^{-1} \boldsymbol{M} \boldsymbol{\tau}^{-1}\right)=\rho\left(h^{-2} k_{i}^{0} k_{j}^{0} \boldsymbol{A}_{i}^{\top} \boldsymbol{M} \boldsymbol{A}_{j}\right),
$$

we may achieve that by forcing every eigenvalue of $\boldsymbol{\tau}^{-1} \boldsymbol{M} \boldsymbol{\tau}^{-1}$ in (25) to be equal to (24). This results in 
the following matrix of stabilization parameters

$$
\begin{gathered}
\boldsymbol{\tau}=\left(\begin{array}{cccc}
\tau_{p} & 0 & 0 & 0 \\
0 & \tau_{\boldsymbol{u}} & 0 & 0 \\
0 & 0 & \tau_{\boldsymbol{u}} & 0 \\
0 & 0 & 0 & \tau_{\boldsymbol{u}}
\end{array}\right) \text { with, } \\
\tau_{p}=\frac{c_{0} h \sqrt{\mu_{\boldsymbol{u}} / \mu_{p}}}{C_{1}\left|\boldsymbol{u}_{d}\right|+c_{0} C_{2}}=\frac{\rho_{0} c_{0}^{2} h}{C_{1}\left|\boldsymbol{u}_{d}\right|+c_{0} C_{2}}, \\
\tau_{\boldsymbol{u}}=\frac{c_{0} h \sqrt{\mu_{p} / \mu_{\boldsymbol{u}}}}{C_{1}\left|\boldsymbol{u}_{d}\right|+c_{0} C_{2}}=\frac{h}{C_{1} \rho_{0}\left|\boldsymbol{u}_{d}\right|+\rho_{0} c_{0} C_{2}},
\end{gathered}
$$

and where use has been made of the expressions for $m_{p}$ and $m_{\boldsymbol{u}}$ in (16). It is observed that:

- The matrix $\boldsymbol{\tau}$ of stabilization parameters (27) is to be inserted into (15) to get the space stabilized variational formulation proposed in this work.

- In the case of a static domain $u_{d}=0$ and we recover from (27) the stabilization parameters for the standard wave equation in mixed form [4],

$$
\tau_{p}=C h \sqrt{\mu_{\boldsymbol{u}} / \mu_{p}} \quad \tau_{\boldsymbol{u}}=C h \sqrt{\mu_{p} / \mu_{\boldsymbol{u}}},
$$

with $C$ standing for a constant. Depending on the values of $\mu_{p}$ and $\mu_{\boldsymbol{u}}$ the mixed wave equation can be used to describe phenomena in classical acoustics, elastodynamics or wave propagation in shallow waters $[4,32]$.
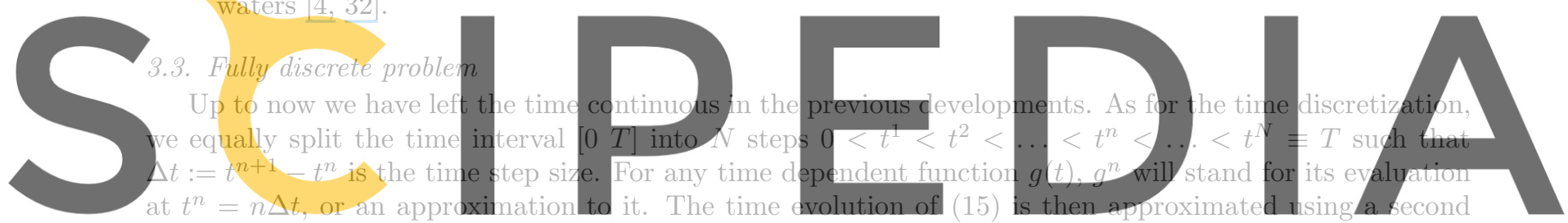

order backward differentiation formula (BDF2). Let us identify $\delta_{t} g^{n+1}:=(1 / 2 \Delta t)\left(3 g^{n+1}-4 g^{n}+g^{n-1}\right)$ so

Register for tree at hiftps/swiwn.scipedia.com to download the version without the watermark $\left(\mu \delta_{t} U_{h}^{n+1}, V_{h}\right)+\left(A_{i} \partial_{i} U_{h}^{n+1}, V_{h}\right)$

$$
+\sum_{e=1}^{n_{e l}}\left(\boldsymbol{A}_{i}^{\top} \partial_{i} \boldsymbol{V}_{h}, \boldsymbol{\tau} \mathcal{P}\left[\left(\delta_{t} \boldsymbol{U}_{h}^{n+1}+\boldsymbol{A}_{i} \partial_{i} \boldsymbol{U}_{h}^{n+1}\right)-\boldsymbol{F}^{n+1}\right]\right)_{\Omega_{e}}=\left(\boldsymbol{F}^{n+1}, \boldsymbol{V}_{h}\right)
$$

If we next focus on the OSS approach, corresponding to the choice $\mathcal{P}=\boldsymbol{I}-\Pi_{h}$, we note that $\mathcal{P}\left(\delta_{t} \boldsymbol{U}_{h}\right)=0$. Then, the expanded fully discrete problem that results from taking into account (8) in (29) and using the BDF2 scheme becomes

$$
\begin{aligned}
& \frac{1}{\rho_{0} c_{0}^{2}}\left(\delta_{t} p_{h}^{n+1}, q_{h}\right)-\frac{1}{\rho_{0} c_{0}^{2}}\left(\boldsymbol{u}_{d} \cdot \nabla p_{h}^{n+1}, q_{h}\right)+\left(\nabla \cdot \boldsymbol{u}_{h}^{n+1}, q_{h}\right) \\
& +\sum_{e=1}^{n_{e l}}\left(\tau_{p} \mathcal{P}\left[-\frac{1}{\rho_{0} c_{0}^{2}} \boldsymbol{u}_{d} \cdot \nabla p_{h}^{n+1}+\nabla \cdot \boldsymbol{u}_{h}^{n+1}-Q^{n+1}\right],-\frac{1}{\rho_{0} c_{0}^{2}} \boldsymbol{u}_{d} \cdot \nabla q_{h}+\nabla \cdot \boldsymbol{v}_{h}\right)_{\Omega_{e}}=\left(Q^{n+1}, q_{h}\right), \\
& \rho_{0}\left(\delta_{t} \boldsymbol{u}_{h}^{n+1}, \boldsymbol{v}_{h}\right)-\rho_{0}\left(\boldsymbol{u}_{d} \cdot \nabla \boldsymbol{u}_{h}^{n+1}, \boldsymbol{v}_{h}\right)+\left(\nabla p_{h}^{n+1}, \boldsymbol{v}_{h}\right) \\
& +\sum_{e=1}^{n_{e l}}\left(\tau_{\boldsymbol{u}} \mathcal{P}\left[-\rho_{0} \boldsymbol{u}_{d} \cdot \nabla \boldsymbol{u}_{h}^{n+1}+\nabla p_{h}^{n+1}-\boldsymbol{f}^{n+1}\right],-\rho_{0} \boldsymbol{u}_{d} \cdot \nabla \boldsymbol{v}_{h}+\nabla q_{h}\right)_{\Omega_{e}}=\left(\boldsymbol{f}^{n+1}, \boldsymbol{v}_{h}\right) .
\end{aligned}
$$

The projection $\mathcal{P}$ is understood to be applied to vectors or scalars, depending on the argument. Equation (29), with the stabilization parameters in (27), is the final fully discrete scheme in space and time proposed in this work, which has been implemented for the numerical examples to be presented in the next section. 


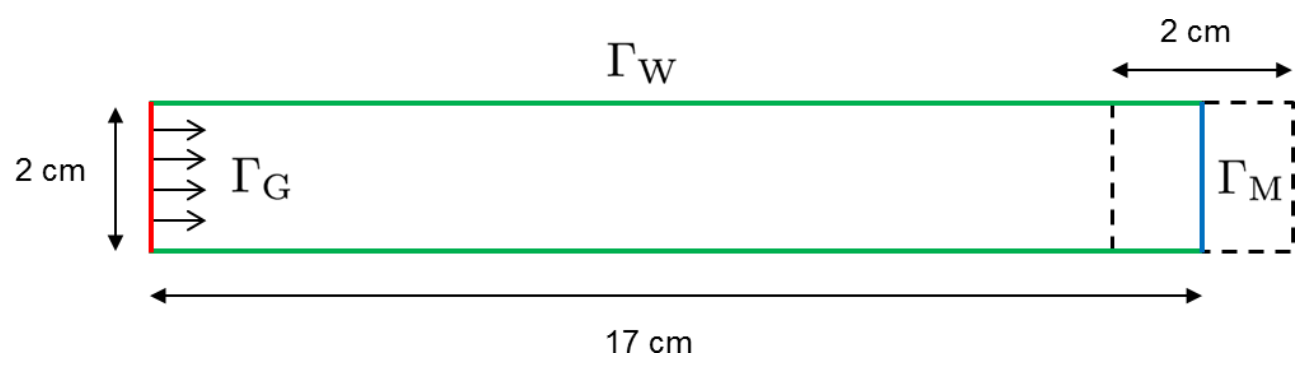

Figure 1: Sketch of the two dimensional duct example used to test the stabilized FEM approach for the ALE mixed wave equation. $\Gamma_{G}$ stands for the boundary where the input signal $\boldsymbol{u}_{g}(t)$ is introduced, $\Gamma_{W}$ the duct walls and $\Gamma_{M}$ the duct exit, which moves sinusoidally backward and forward.

\subsection{Motion of the computational mesh}

With regard to the motion of the computational mesh, it will be prescribed by the known displacement at the domain boundaries. The motion of the boundary nodes becomes smoothly transmitted to the inner mesh nodes through diffusion, i.e. using the standard strategy of solving the Laplacian equation for the node displacements $\boldsymbol{w}(\boldsymbol{x}, t)$. Therefore, at each time step of (29) we will solve the additional equation

$$
\nabla^{2} w^{n+1}=0
$$

with boundary conditions
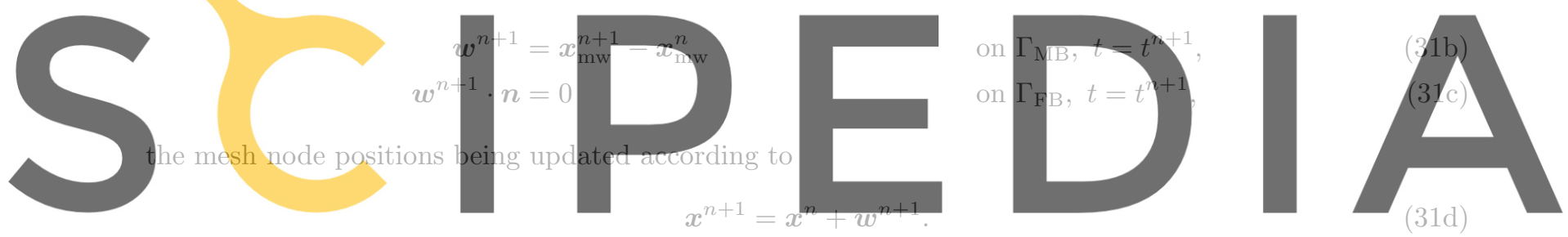

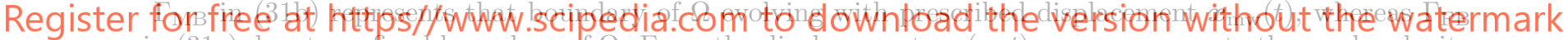 in (31c) denotes a fixed boundary of $\Omega$. From the displacements $w(x, t)$ one can compute the mesh velocity using an appropriate time integration scheme.}

\section{Numerical examples}

\subsection{Two dimensional duct with periodically varying length}

In order to check the performance of the proposed stabilization strategy, we have adopted a numerical example consisting of a two dimensional duct of constant cross section but varying length, see Fig. 1. A periodic velocity $\boldsymbol{u}_{g}(t)$ has been imposed at the duct entrance $\left(\Gamma_{G}\right.$ in the figure) which results in the generation of plane waves propagating to the opposite side of the duct. Yet, a periodic displacement of different frequency has been imposed at the exit boundary $\Gamma_{M}$. This guarantees that the mesh velocity $\boldsymbol{u}_{d}$ will be parallel to the acoustic pressure gradient. When $\boldsymbol{u}_{d}$ is parallel to $\nabla p$, the importance of the ALE terms in (4) becomes maximum so the test can be seen as demanding for the validation of the stabilized method. Besides, note that all duct dimensions, boundary displacements and involved frequencies (see below) have been chosen to be of the same order of that found in real human vocal tracts, lip protrusion movements and formant frequencies. This will guarantee that stabilization will perform correctly when addressing the production of diphthongs.

The details of the simulation are as follows. We have split the duct boundary into three non-intersecting regions $\Gamma_{\mathrm{G}}, \Gamma_{\mathrm{W}}$ and $\Gamma_{\mathrm{M}}$ (see Fig. 1) and thus supplement the ALE mixed wave equation (4) with boundary 


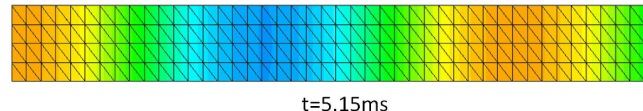

$\mathrm{t}=5.15 \mathrm{~ms}$

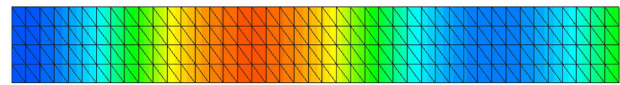

$\mathrm{t}=12.775 \mathrm{~ms}$

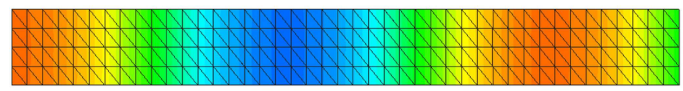

$\mathrm{t}=23.45 \mathrm{~ms}$

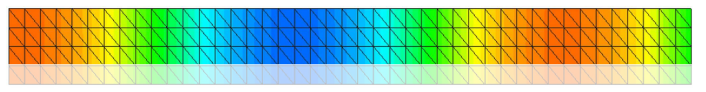

$t=25.8 \mathrm{~ms}$
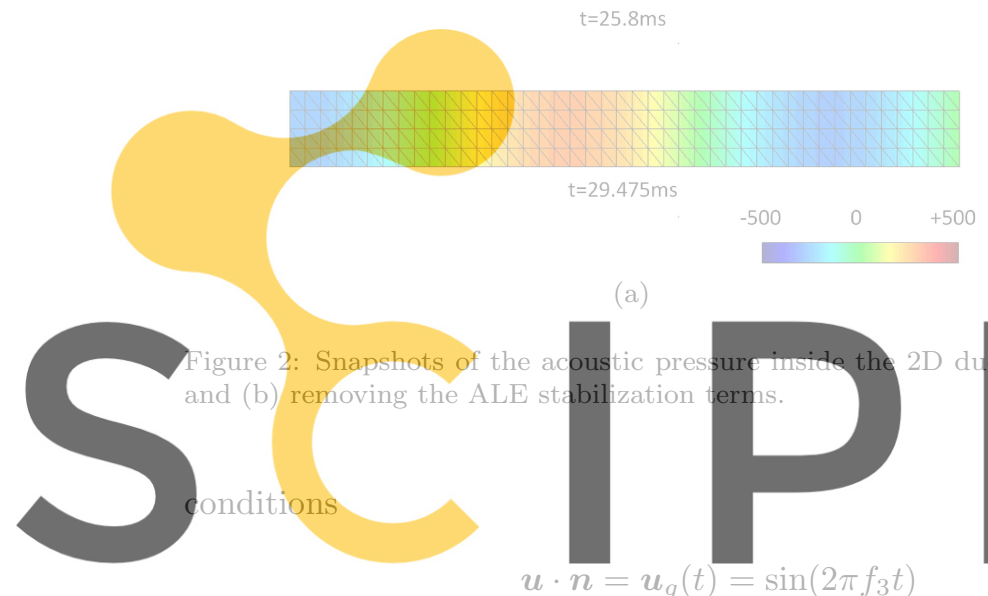

$\boldsymbol{u} \cdot \boldsymbol{n}=\boldsymbol{u}_{g}(t)=\sin \left(2 \pi f_{3} t\right)$

Register for free at https $\mu \cdot n=0$ $p=0$
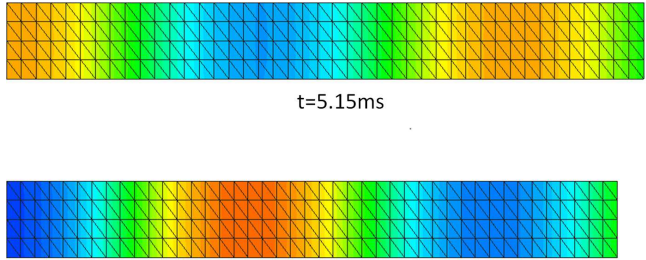

$\mathrm{t}=12.775 \mathrm{~ms}$

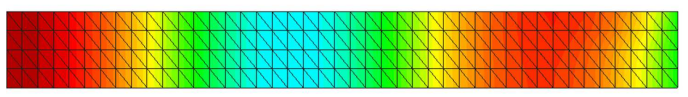

$\mathrm{t}=23.45 \mathrm{~ms}$

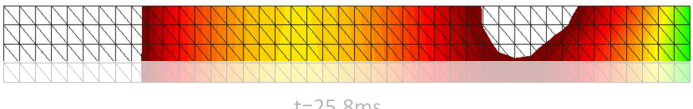

$\mathrm{t}=25.8 \mathrm{~ms}$

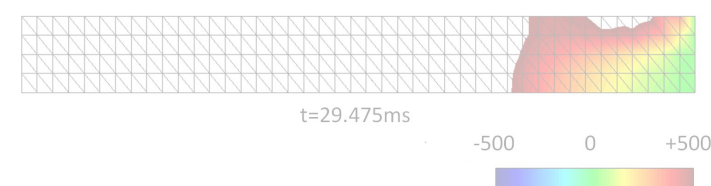

(b)
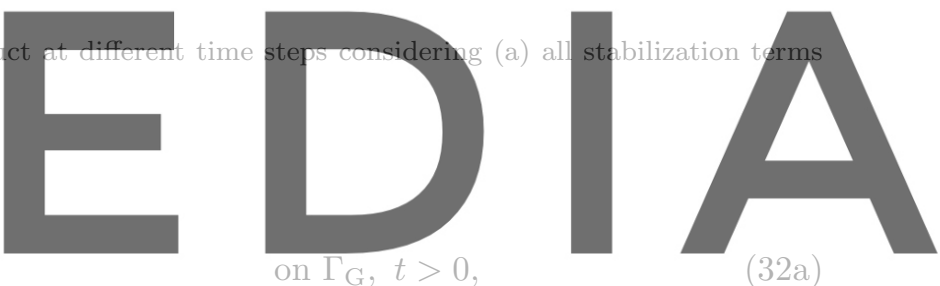

(32a)

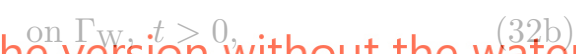
on $\Gamma_{\mathrm{M}}, t>0$.

It is observed that we have assumed rigid boundaries and impose a zero pressure release condition at the duct exit. $\boldsymbol{n}$ denotes the normal pointing outwards the computational boundary. With regard to the velocity on $\Gamma_{G}$, it has a sinusoidal time dependence whose frequency corresponds to the third resonance mode of the mean length duct $(L=17 \mathrm{~cm})$. The $n$-th resonance frequency of such a duct is given by $f_{n}=(2 n-1) c_{0} /(4 L)$ so by taking $n=3, c_{0}=350 \mathrm{~m} / \mathrm{s}$ and $L=17 \mathrm{~cm}$ we get $f_{3} \simeq 2573 \mathrm{~Hz}$. A periodic displacement of frequency $f_{M}=500 \mathrm{~Hz}$ has been imposed for $\Gamma_{M}$. Finally, zero pressure and zero particle velocity have been considered for $t=0$ inside the duct.

The simulations have been carried out using the fully discrete scheme in (29), activating and deactivating the ALE stabilizations terms, and using a sampling frequency rate of $f_{s}=1 / \Delta t=80 \mathrm{kHz}$. From numerical experiments, the values deemed appropriate for the constants $C_{1}$ and $C_{2}$ in the expression for the stabilization parameters (27) are $C_{1}=C_{2}=0.01$. The obtained results are presented in Fig. 2 . In the first column we show different snapshots of the acoustic pressure inside the duct when using all stabilization terms. As observed, the inner pressure pattern basically corresponds to the third acoustic mode distribution which does not become much altered by the movement of the exit boundary. The solution is stable and does not change significantly as time evolves. This is not the case if the ALE stabilization terms are removed from the formulation, as can be readily appreciated in the right column of Fig. 2. Though the solution matches the correct one for the initial time steps of the simulation, as long as time evolves the solution becomes unstable and finally blows up, totally exceeding the plotted range $[-500,500] \mathrm{Pa}$. 
la/
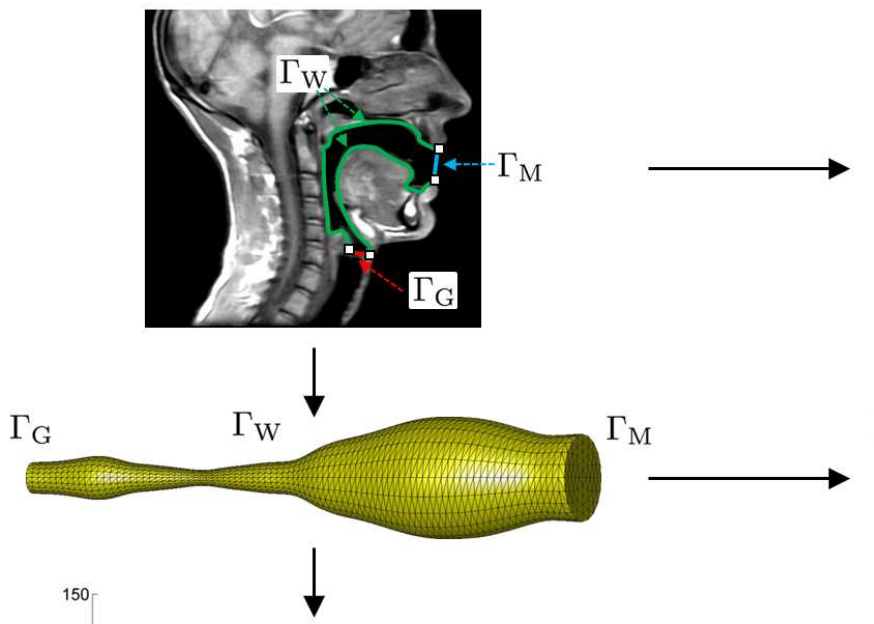

140

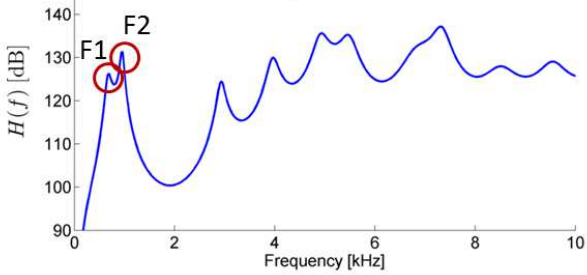

/i/
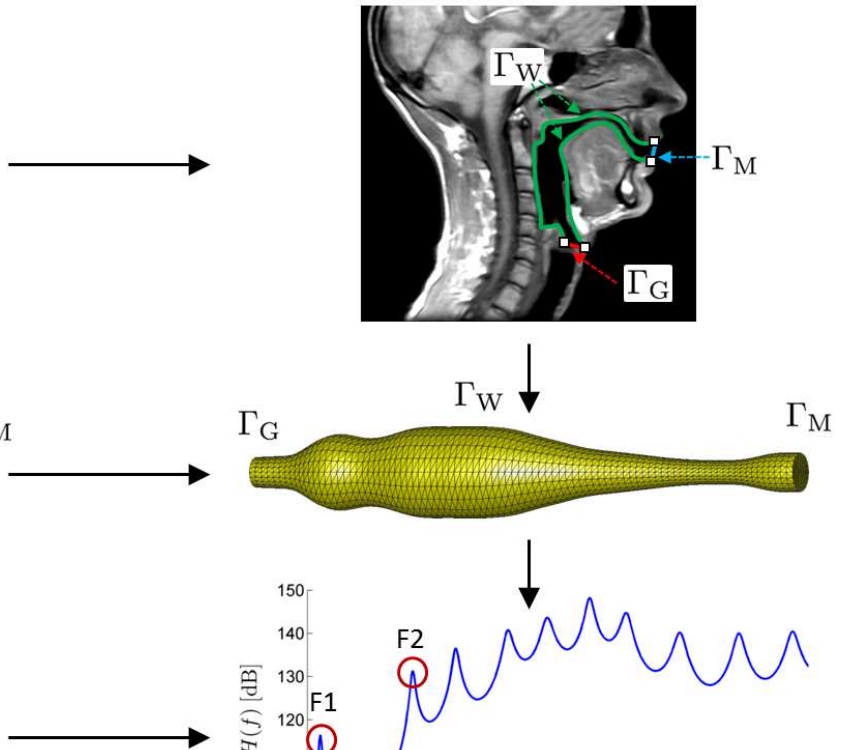

Figure 3: Sketch for the numerical production of vowels. From magnetic resonance imaging (MRI), data is obtained for the computation of vocal tract transfer functions (VTTF). The left column corresponds to vowel $/ \mathrm{a} /$ and the right column to vowel /i/. The diphthong /ai/ involves transiting from /a/ to /i/.

\subsection{Production of diphthongs in $3 D$}

A more applied example related to voice production will be next addressed. In particular, we will focus on the numerical generation of diphthongs. The diphthong /ai/ will be used for illustrative purposes though the presented procedure has general validity.

Let us start by reviewing how a single vowel could be generated using numerical methods. In a nutshell, to produce say, vowel /a/, we could proceed as follows (see left column in Fig. 3). First a magnetic resonance imaging (MRI) is taken from the vocal tract of a given individual when pronouncing the sound /a/. Though nowadays very detailed 3D geometries can be obtained [36], it is not always necessary to use such intricate shapes for voice generation. Actually we can neglect the influence of some of the vocal tract cavities, such as the valleculae or the piriform fossae, and work with simplified straight vocal tracts with radial symmetry based on real vocal tract cross sections, as those in [37] (see second row in the first column of Fig. 3). Despite of its simplicity, fairly good results can still be obtained with these smoothed vocal tract geometries. Since it is not the goal of this work to reproduce diphthongs from a given individual with high fidelity, but rather to propose a general approach for diphthong generation, we will make use of these simplified geometries in the simulations to be presented below. Next, we just need to solve the wave equation for the acoustic pressure inside such vocal tracts supplemented with appropriate boundary conditions; a model for the glottal pulses generated by the vocal chords has to be input at the glottis area $\left(\Gamma_{G}\right.$ in Fig. 3), and impedance and radiation conditions have to be respectively specified at the vocal tract walls $\Gamma_{W}$ and mouth exit $\Gamma_{M}$, which are indicated later. Finally, collecting the time evolution of the acoustic pressure at a node close to the exit of the mouth and converting its values into an audio file, we will produce the sound of an /a/. It is to be noted that vowels are actually generated by acoustic resonance excitation of the air volume inside the vocal tract. The eigenfrequencies of the air resonating inside the vocal tract, which are known as formants in the voice and speech communities, will change from one vowel to another. Formants can be clearly appreciated 


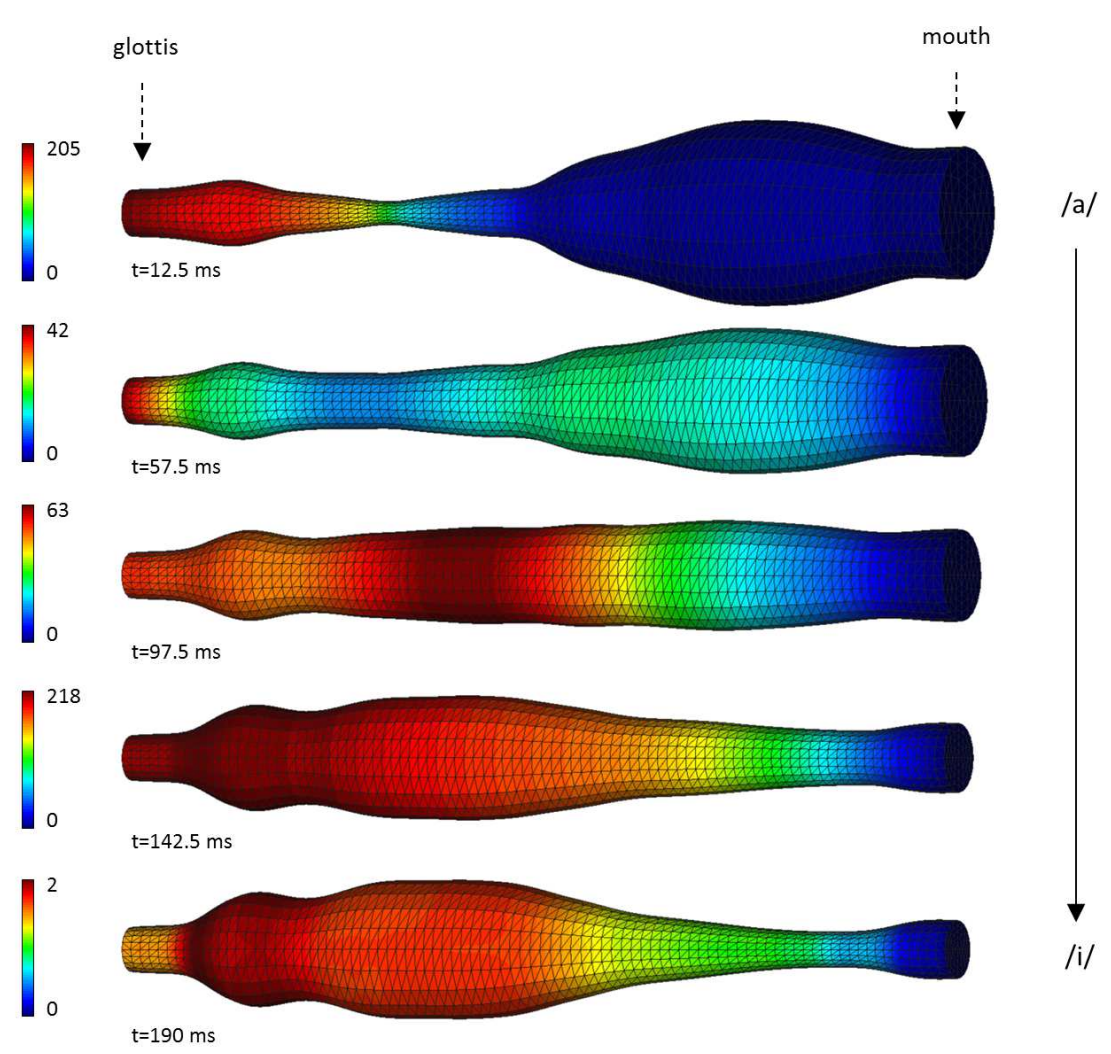

Figure 4: Snapshots at five different time instants showing the transition from vowel /a/ to /i/. The acoustic pressure at the boundaries of the vocal tract and its shape evolution can be appreciated.

as peaks in the so called vocal tract transfer functions (VTTF). These are computed from the quotient between the Fourier transform of the acoustic pressure at a node located close to the mouth exit, and the Fourier transform of the volume velocity corresponding to the glottal pulses being input at $\Gamma_{G}$. A VTTF for vowel /a/ is shown in the last row of the left column of Fig. 3. The first two formants F1 and F2 have been highlighted as those are the ones which allow listening individuals to distinguish between vowel sounds

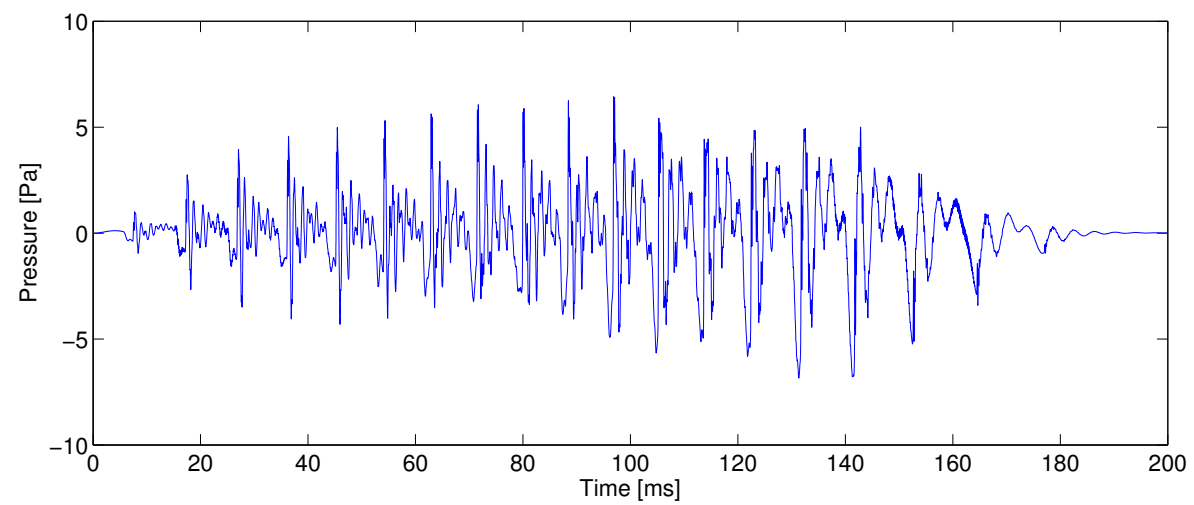

Figure 5: Time evolution of the acoustic pressure at a point close to the mouth exit. When converted to audio, it produces the sound /ai/. 


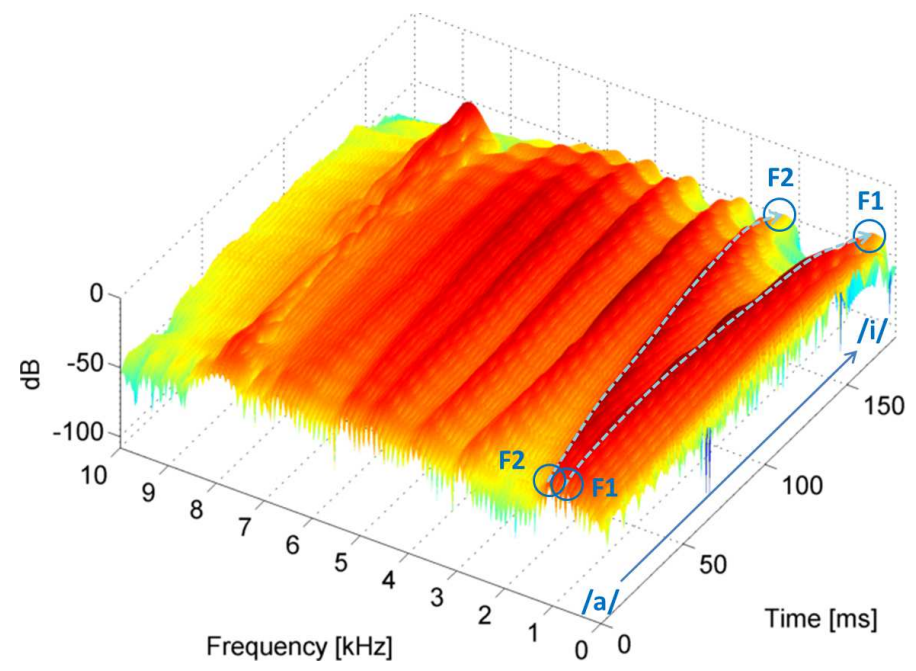

Figure 6: Spectrogram of the acoustic pressure at the node located close to mouth exit from Fig. 5. Formant trajectories from /a/ to /i/ have been highlighted.

(compare with the VTTF corresponding to an /i/ in the last row of the right column in Fig. 3). Higher formants are responsible for other vowel sound finer features like timber.

If we next focus on the generation of the diphthong /ai/, it is clear that we will have to transition from the situation in the left column in Fig. 3 to the one in the right column. Some simplifications have been assumed. First, a linear interpolation from the geometry of the vocal tract of vowel /a/ to the vocal tract of vowel /i/ has been performed, though more accurate options exist, based on controlling the formant transition trajectories between vowels [38]. Second, we have simply imposed a zero pressure release condition $p=0$ at the mouth exit $\Gamma_{M}$. This is a rough approximation to emulate radiation losses, which is known to shift the formants towards upper frequencies depending on the formant-cavity affiliation phenomenon [39, 40], as well as to decrease the formant bandwidths for mid-high frequencies (see e.g., [14] for a detailed discussion). A more precise option would consist in allowing acoustic waves to emanate from the mouth and radiate towards infinity. This would involve, for example, implementing a perfectly matched layer (PML) $[41,42,13]$ for the finite element approximation of the ALE wave equation presented in this work, or imposing a good enough performing absorbing boundary condition (see e.g., [? 43]). This has been left for future developments.

To produce the diphthong /ai/ we have solved the ALE mixed wave equation (4) using the stabilized FEM approach described in the preceding Section 3, in the moving computational domain consisting of the transition from the vocal tract of /a/ in the left column of Fig. 3, to the geometry of /i/ in the right column. The following conditions have been imposed on the vocal tract boundaries

$$
\begin{aligned}
\boldsymbol{u} \cdot \boldsymbol{n} & =\boldsymbol{u}_{g}(t) & & \text { on } \Gamma_{\mathrm{G}}, t>0, \\
\boldsymbol{u} \cdot \boldsymbol{n} & =p / Z_{w} & & \text { on } \Gamma_{\mathrm{W}}, t>0, \\
& p=0 & & \text { on } \Gamma_{\mathrm{M}}, t>0 .
\end{aligned}
$$

The boundary velocity $\boldsymbol{u}_{g}(t)$ in (33a) stands for a train of glottal pulses generated using a model of the Rosenberg type [44]. In order to increase the naturalness of the produced diphthong these pulses have been enhanced introducing a pitch curve for the fundamental frequency, adding some shimmer to the glottal pulse amplitudes and also applying a fade in/out to the global signal. In (33b), $Z_{w}$ stands for the wall impedance, which is related to the wall admittance coefficient, $\mu$, by $\mu=Z_{0} / Z_{w}, Z_{0}=\rho_{0} c_{0}$ being the characteristic acoustic impedance. We have taken the standard value $\mu=0.005$ which fairly approximates losses of vocal tract tissues [10]. Zero initial values have been taken for the acoustic pressure and acoustic particle velocity.

The moving vocal tract domain has been initially meshed using a structured tetrahedral mesh with initial element size $h \simeq 0.002 \mathrm{~m}$ and evolved according to the procedure described in Subsection 3.4. The 
simulation has last for $200 \mathrm{~ms}$ and a sampling rate of $f_{s}=1 / \Delta t=80 \mathrm{kHz}$ has been used. The standard value in vocal tract acoustics of $c_{0}=350 \mathrm{~m} / \mathrm{s}$ has been used for the sound speed. Again, we have used the values $C_{1}=C_{2}=0.01$ for the constants in the stabilization parameters (27).

In Fig. 4, a sequence of five snapshots corresponding to the transition from vowel /a/ to / i/ is presented. The changes in vocal tract shape can be clearly appreciated. The first snapshot at $t=12.5 \mathrm{~ms}$ corresponds to the articulation of an /a/, whereas the last one at $t=190 \mathrm{~ms}$ corresponds to that of an /i/. The acoustic pressure values at the vocal tract boundaries for the selected time instants can be also appreciated. Different limiting values have been chosen for the color scales to better perceive the acoustic pressure distribution at the vocal tract for a particular time instant. In Fig. 5 we show the time evolution for the acoustic pressure at a point close to the mouth exit. When transforming this plot into an audio file we get, as expected, the sound /ai/. Finally, in Fig. 6 we show the spectrogram corresponding to the acoustic pressure in Fig. 5. This has been computed using a hamming window with a frame width of $20 \mathrm{~ms}$ and an overlap of $1 \mathrm{~ms}$. As usually done in speech processing, a pre-emphasis FIR filter with coefficients [1 -0.97] has been applied to enhance the visualization of the high frequency range. In the first curve of the spectrogram, corresponding to vowel /a/, we can clearly identify the two formants previously highlighted in the VTTF of /a/, in the last row of the left column of Fig. 3. Similarly, the last curve of the spectrogram corresponds to vowel /i/, and the two formants identified in the VTTF of /i/ (last row of the right column of Fig. 3) can also be clearly recognized. The trajectories of the two formants F1 and F2, which separate apart from /a/ to /i/, have been indicated in Fig. 6 to better show their evolution.

\section{Conclusions}

A stabilized finite element method (FEM) has been proposed to solve the wave equation in mixed form in an ALE frame of reference. The method is based on the subgrid scale approach, and its good performance strongly relies on the proposed design for the matrix of stabilization parameters. The stabilized FEM allows us to use equal interpolations for the acoustic pressure and acoustic particle velocity, avoiding any locking effect. This has been tested by means of a two dimensional benchmark problem consisting of wave propagation inside a duct with a moving exit boundary.

On the other hand, the proposed approach has been applied to voice production, in particular to the generation of diphthongs. A general procedure has been presented for that purpose and diphthong /ai/ has been used as an example. Fairly good and promising results have been obtained. Future work will involve dealing with more realistic vocal tract geometries and permitting acoustic waves to emanate from the mouth and propagate outwards, to get more accurate solutions.

\section{Acknowledgments}

This work is supported by EU-FET grant EUNISON 308874. The second author also acknowledges the Generalitat de Catalunya (SUR/ECO) for the pre-doctoral FI Grant No. 2013FI - B1 00165 and the third author also gratefully acknowledges the support received from the Catalan Government through the ICREA Acadèmia Research. The fourth author is grateful to the Ministerio de Educación, Cultura y Deporte, Programa de Formación de Profesorado Universitario (FPU), grant No. AP2010 - 0563.

\section{References}

[1] F. Fahy, Sound Intensity, E \& FN Spon, London, UK, 1995.

[2] M. L. Munjal, Acoustics of Ducts and Mufflers, Wiley, New York, 1987.

[3] L. E. Kinsler, A. R. Frey, A. B. Coppens, J. V. Sanders, Fundamentals of Acoustics, 4th Edition, Wiley, New York, 2000.

[4] R. Codina, Finite element approximation of the hyperbolic wave equation in mixed form, Comput. Methods Appl. Mech. Engrg.197(13-16) (2008) 1305-1322.

[5] W. Zhao, C. Zhang, S. Frankel, L. Mongeau, Computational aeroacoustics of phonation, part i: Computational methods and sound generation mechanisms, J. Acoust. Soc. Am.112(5) (2002) 2134-2146.

[6] W. Zhao, C. Zhang, S. Frankel, L. Mongeau, Computational aeroacoustics of phonation, part ii: Effects of flow parameters and ventricular folds, J. Acoust. Soc. Am.112(5) (2002) 2147-2154. 
[7] G. Link, M. Kaltenbacher, M. Breuer, M. Döllinger, A 2D finite-element scheme for fluid-solid-acoustic interactions and its application to human phonation, Comput. Methods Appl. Mech. Engrg.198(41-44) (2009) 3321-3334.

[8] H. Luo, R. Mittal, X. Zheng, S. A. Bielamowicz, R. J. Walsh, J. K. Hahn, An immersed-boundary method for flow-structure interaction in biological systems with application to phonation, J. Comput. Phys.227 (22) (2008) 9303 - 9332.

[9] F.-B. Tian, H. Dai, H. Luo, J. F. Doyle, B. Rousseau, Fluid-structure interaction involving large deformations: 3d simulations and applications to biological systems, J. Comput. Phys.258 (0) (2014) $451-469$.

[10] P. Švancara, J. Horáček, Numerical modelling of effect of tonsillectomy on production of czech vowels, Acta Acust.92 (2006) 681-688.

[11] T. Vampola, J. Horáček, J. G. Švec, FE modeling of human vocal tract acoustics. Part I: Production of czech vowels, Acta Acust.94(5) (2008) 433-447.

[12] T. Vampola, A. M. Laukkanen, J. Horáček, J. G. Švec, Vocal tract changes caused by phonation into a tube: a case study using computer tomography and finite-element modeling, J. Acoust. Soc. Am.129(1) (2011) 310-315.

[13] M. Arnela, O. Guasch, Finite element computation of elliptical vocal tract impedances using the two-microphone transfer function method, J. Acoust. Soc. Am.133(6) (2013) 4197-4209.

[14] M. Arnela, O. Guasch, F. Alías, Effects of head geometry simplifications on acoustic radiation of vowel sounds based on time-domain finite-element simulations, J. Acoust. Soc. Am.134(4) (2013) 2946-2954.

[15] H. Matsuzaki, N. Miki, Y. Ogawa, 3D finite element analysis of japanese vowels in elliptic sound tube model, Electron. Comm. Jpn. 3 83(4) (2000) 43-51.

[16] K. Motoki, Three-dimensional acoustic field in vocal-tract, Acoust. Sci. \& Tech.23(4) (2002) 207-212.

[17] T. Kako, K. Touda, Numerical method for voice generation problem based on finite element method, J. Comput. Acoust.14(1) (2006) 45-56.

[18] A. Hannukainen, T. Lukkari, J. Malinen, P. Palo, Vowel formants from the wave equation, J. Acoust. Soc. Am.122(1) (2007) EL1-EL7.

[19] H. Takemoto, P. Mokhtari, T. Kitamura, Acoustic analysis of the vocal tract during vowel production by finite-difference time-domain method, J. Acoust. Soc. Am.128(6) (2010) 3724-3738.

[20] T. Hughes, W. Liu, T. Zimmermann, Lagrangian-eulerian finite-element formulation for compressible viscous flows, Comput. Methods Appl. Mech. Engrg.29 (1981) 329-349.

[21] A. Huerta, W. Liu, Viscous flow with large free surface motion, Comput. Methods Appl. Mech. Engrg.69 (1988) $277-324$.

[22] E. Bécache, P. Joly, C. Tsogka, An analysis of new mixed finite elements for the approximation of wave propagation problems, SIAM J. Numer. Anal.37(4) (2000) 1053-1084.

[23] E. Bécache, P. Joly, C. Tsogka, Fictitious domains, mixed finite elements and perfectly matched layers for 2-D elastic wave propagation, J. Comput. Acoust.9(3) (2001) 1175-1201.

[24] E. Bécache, P. Joly, C. Tsogka, A new family of mixed finite elements for the linear elastodynamic problem, SIAM J. Numer. Anal.39(6) (2002) 2109-2132.

[25] T. Hughes, Multiscale phenomena: Green's function, the dirichlet-to-neumann formulation, subgrid scale models, bubbles and the origins of stabilized formulations, Comput. Methods Appl. Mech. Engrg.127 (1995) 387-401.

[26] T. Hughes, G. Feijóo, L. Mazzei, J. Quincy, The variational multiscale method, a paradigm for computational mechanics, Comput. Methods Appl. Mech. Engrg.166 (1998) 3-24.

[27] R. Codina, Stabilized finite element approximation of transient incompressible flows using orthogonal subscales, Comput. Methods Appl. Mech. Engrg.191 (2002) 4295-4321.

[28] S. Badia, R. Codina, H. Espinoza, Stability, convergence and accuracy of stabilized finite elements methods for the wave equation in mixed form, SIAM J. Numer. Anal.52 (2014) 1729-1752.

[29] H. P. Langtangen, G. Pedersen, Computational models for weakly dispersive nonlinear water waves, Comput. Methods Appl. Mech. Engrg.160 (3-4) (1998) 337-358.

[30] M. B. M. Walkley and, A finite element method for the two-dimensional extended Boussinesq equations, Int. J. Numer. Meth. Fluids39 (10) (2002) 865-885.

[31] G. Hauke, A symmetric formulation for computing transient shallow water flows, Comput. Methods Appl. Mech. Engrg.163 (1-4) (1998) 111-122.

[32] R. Codina, J. González-Ondina, G. Díaz-Hernández, J. Principe, Finite element approximation of the modified boussinesq equations using a stabilized formulation, Int. J. Numer. Meth. Fluids57(9) (2008) 1249-1268.

[33] A. Huang, R. Temam, The linearized 2D inviscid shallow water equations in a rectangle: boundary conditions and wellposedness, Arch. Rational Mech. Anal.211 (2014) 1027-1063.

[34] J. Donea, A. Huerta, J.-P. Ponthot, A. Rodríguez-Ferran, Arbitrary Lagrangian Eulerian Methods, in: Encyclopedia of Computational Mechanics, John Wiley \& Sons, Ltd, 2004.

[35] R. Codina, J. Principe, O. Guasch, S. Badia, Time dependent subscsales in the stabilized finite element approximation of incompressible flow problems, Comput. Methods Appl. Mech. Engrg.196(21-24) (2007) 2413-2430.

[36] D. Aalto, O. Aaltonen, R.-P. Happonen, P. Jääsaari, A. Kivelä, J. Kuortti, J.-M. Luukinen, J. Malinen, T. Murtola, R. Parkkola, J. Saunavaara, T. Soukka, M. Vainio, Large scale data acquisition of simultaneous $\{$ MRI $\}$ and speech, Appl. Acoust.83 (0) (2014) 64-75.

[37] B. H. Story, Comparison of magnetic resonance imaging-based vocal tract area functions obtained from the same speaker in 1994 and 2002, J. Acoust. Soc. Am.123(1) (2008) 327-335.

[38] B. H. Story, A parametric model of the vocal tract area function for vowel and consonant simulation, J. Acoust. Soc. Am.117(5) (2005) 3231-3254.

[39] G. Fant, Acoustic Theory of Speech Production, 2nd Edition, Mouton, Paris, 1970.

[40] K. N. Stevens, Acoustic phonetics, The MIT Press, Cambridge, MA, 2000. 
[41] J. Berenger, A perfectly matched layer for the absorption of electromagnetic waves, J. Comput. Phys.114 (2) (1994) 185-200.

[42] B. Kaltenbacher, M. Kaltenbacher, I. Sim, A modified and stable version of a perfectly matched layer technique for the 3-d second order wave equation in time domain with an application to aeroacoustics, J. Comput. Phys.235 (2013) 407-422.

[43] H. Espinoza, R. Codina, S. Badia, A Sommerfeld non-reflecting boundary condition for the wave equation in mixed form, Comput. Methods Appl. Mech. Engrg.276 (2014) 122-148.

[44] A. E. Rosenberg, Effect of glottal pulse shape on the quality of natural vowels, J. Acoust. Soc. Am.49(2) (1971) 583-590. 